(RESEARCH ARTICLE)

\title{
COVID-19; evolving of triage, management protocol and maternal health in Islamic Republic of Iran
}

Alireza Raeisi 1,2, Nasrin Changizi 1, 3, ${ }^{*}$, Hamed Barekati 1, Leila Hadipour Jahromy 1, Saeideh Hejazi 1, Susan Rahimi Ghasabeh ${ }^{1}$, Zahra Farahani ${ }^{3}$ and Raheleh Moradi ${ }^{3}$

${ }^{1}$ Iran Ministry of Health and Medical Education, Tehran, Iran.

${ }^{2}$ Shiraz University of Medical Sciences, Shiraz, Iran.

${ }^{3}$ Maternal, Fetal and Neonatal Research Center, Tehran University of Medical Sciences Tehran, Iran.

Publication history: Received on 26 July 2020; revised on 17 August 2020; accepted on 20 August 2020

Article DOI: https://doi.org/10.30574/wjarr.2020.7.3.0279

\begin{abstract}
National Maternal Health program is based over multidisciplinary evidence-based infrastructure, which in 2017 was reported to be on track of sustainable development goals by World Health Organization. With epidemic distribution of COVID-19, maternal health program was among the first health programs for evidence-based protocol preparation in Islamic Republic of Iran at the national level. Usually in this national program, Obstetrical protocols updating takes place at most every 2-3 years but due to paucity of evidences at first and then rapid evolving conditions of the COVID-19, these changes are done nearly on a monthly basis (in this regard triage system and multiple additional protocols were sent to the associated fields). On $1^{\text {st }}$ of March, we designed the Triage system for COVID-19 in pregnancy. Since there were paucity of data and documentation in this regard, we used adult triage flow chart as the prototype and adapted it to pregnancy situation. Then based on the more evidences and more and more experiences, the triage system and flow chart were updated and now we had reached the 4th version now. Due to rapid release of new studies and evidences as well as national experiences and needs, protocols associated with management of pregnancy and COVID -19 are updating rapidly.
\end{abstract}

Keywords: COVID-19; Triage; Management; Maternal health

\section{Introduction}

Coronavirus Disease 2019 (COVID-19) as a severe and acute disease with a rapid spread was firstly identified in Wuhan, China, in December 2019. Now COVID-19 is known the most infectious diseases worldwide and World Health Organization (WHO) declared it as a global public health emergency [1-3].

Clinical symptoms associated with COVID-19 present from a mild flu-like symptom to pneumonia, ARDS, and multiorgan failure [2]. This infectious disease can lead to a rapid increase in morbidity and mortality rates [1]. Several published studies are showing epidemiological data and clinical outcomes associated with COVID-19 among the nonpregnant population. There are very limited investigations assessing the pregnancy and probability of vertical transmission of COVID-19 through pregnancy and delivery. Previous studies demonstrated that different compromising alterations in immunological/ physiological responses of the body during pregnancy may impose a pregnant woman to a greater risk of COVID-19 infection [4, 2].

It was reported that symptoms related to COVID-19 in pregnant women are similar to those in non-pregnant adults'; fever and cough are the main manifestations by 80\% [1]. Although no vertical transmission of COVID-19 was reported by several studies, its possibility cannot be ruled out [5]. Similarities in structures and pathogenesis of both SARS-CoV-

\footnotetext{
${ }^{*}$ Corresponding author: Nasrin Changizi

Iran Ministry of Health and Medical Education, Tehran, Iran.
} 
1 and COVID-19 may lead to a similarity in the characteristic of a low potential risk of vertical transmission [4, 6]; however, 2 recent studies from Italy and France (July 2020) could show the presence of the virus in the placental tissue and nasal swabs of neonates born from infected pregnant women with the SARS-CoV-2 coronavirus [7, 8]. With respect to the influence of COVID-19 on perinatal outcome an investigation from Iran (2020) has indicated that through a 30day period, of 9 pregnant women affected by COVID-19, 7 cases died and a case was critically ill and ventilator dependent [9]. A maternal death due to COVID-19 was also reported in United Kingdom in 2020; a 29-year old diabetic pregnant woman was hospitalized in the intensive care unit but she died with diagnosis of pulmonary embolism and cerebral basilar artery thrombosis [10]. The results of a systematic review and meta-analysis have demonstrated that despite no vertical transmission, the incidence rates related to preterm birth $(41.1 \%)$ and perinatal death $(7.0 \%)$ were notable among COVID-19 infected mothers [11]. According to Royal College of Obstetricians \& Gynaecologists (RCOG) guidance, there is no evidence for restriction of vaginal delivery or preferred anesthesia in mothers with COVID-19; however, the route of Childbirth and type of anesthesia should be individualized based on obstetrical as well as general maternal health status $[5,12]$. A former study suggested several managerial principles regarding prenatal COVID-19 exposure including early isolation, infection control procedures and antibiotic therapy, testing for SARS-CoV-2 and coinfection, oxygen therapy, avoidance of fluid imbalance, monitoring of fetal heart rate and uterine contraction, intubation and mechanical ventilation, individualized childbirth planning, and multi-disciplinary management [1].

Outbreak of covid-19 was announced as a pandemic by the WHO. The primary responsibility of governments is to ensure the provision of the best available health services for the community. Establishing sustainable development based on health measures should be considered by all governmental and non-governmental departments.

\section{Methods}

In Islamic Republic of Iran as a country for being on track of Sustainable Development Goals (SDG) in maternal health program, we acted meticulously about implementing management protocols from the early days. So with early warning signs in the country, the maternal health programs were focused specially on the management of COVID -19 during pregnancy and post-partum.

While first recommendation on COVID -19 in pregnancy was published on $25^{\text {th }}$ of February, COVID -19 in pregnancy triage flowchart and protocol became on Board from 2020.03.03 by Ministry of Health and Medical Education (MOHME) in collaborations with National Maternal Health Committee and after approval of Corona Virus Scientific Committee in MOHME. This guideline constitutes a Triage flowchart approaching both respiratory as well as obstetrical status of the pregnant mothers, practically adapted approaches for both inpatient and outpatient care in pregnancy. But due to the rapid evolving characteristics of COVID-19 (in contrast to usual protocol writing practices in maternal health program in our office, which usually takes at least 2-3 years for next updating), this specific protocol is changing nearly every 46 weeks based on the need assessments and also new worldwide evidences.

\section{Results and discussion}

In addition to the changes in triage flowchart, we published multiple protocols based on the need in the past few months as mentioned in Table 1 and Fig. 1. Main changes in less than recent 4 months have been as following:

\subsection{First edition of protocols was published on 2020.02 .25}

A protocol was published based on the sparse evidence from Chinese experiences 25th February, which include multiple Dos and Don'ts regarding to pregnancy/postpartum course and Covid-19. In this protocol we planned for following actions through the medical universities:

Pathway of diagnosis and management of cases

Pathway of transferring of mothers

Formation of Medical Universities Maternal Health Committees including Obstetrician/Pediatrician/Anesthesiologist Recommendation for how to decide for termination of pregnancy in infected /suspected cases Recommendation for mass media education.

\subsubsection{First version of flowchart was released on 2020.03 .03}

- $\quad$ The simple Pathway of diagnosis and management of the pregnant-postpartum mothers suspected for COVID19 was distributed. 

below this level.

Lower limit of 02 Saturation was set on 93rd percent and the protocol of hospitalization was planned for cases

- Pharmacologic protocols were designed based on 2 approaches:

1- The first one was limited to 2 drug approaches: Oseltamivir with Hydroxy Cholorquine or Chloroquine Phosphate.

2- The second One included Oseltamivir with Hydroxy Cholorquine or Chloroquine Phosphate with Lopinavir/Ritonavir.

\subsection{Second version of flowchart was released on 2020.03 .20}

3.2.1. For the honor of rapid diagnosis and management of the confirmed/suspected pregnant cases in the respiratory triage, low dose HRCT as a diagnostic modality was proposed for all pregnant women once the diagnostic approach becomes necessary (Low Dose HRCT System set up protocol was attached to the protocol). In this regard the following recommendations were:

For pregnant women preferably imaging procedures were scheduled at a specific time and apart from other patients.

Once becoming ready for imaging with the standard personal protective equipment, they will go through the imaging procedure.

- Use of a standard antiseptic agent to disinfect contact surfaces after each procedure for every infected or suspicious subject.

- $\quad$ Using an apron shield was necessary for the protection of fetuses against radiation exposure

- $\quad$ In case of lack of access to Low Dose HRCT, after stabilizing the pregnant mother (based on the consultation with university Maternal Health advisory committee), a Chest radiography examination will be performed or the patient will be referred to a CT scan equipped center. Routine repeat imaging is abandoned unless prescribed based on individualized indications in some patients.

\subsubsection{Laboratory tests assessing viral infection (including PCR) were requested once the patient hospitalized.}

3.2.3. Medical treatments of COVID-19 for infected pregnant women were designed based on the groups; outpatient infected pregnant women and hospitalized infected pregnant women.

Different therapeutic teams were designed for pregnant women in the hospital. The first one was the outpatient consultancy team which included Obstetrician/Gynecologist and Infectious diseases specialist who were responsible for outpatient cases evaluation and deciding on their platform of care (hospitalization/Home care) as well as responding to Health centers consultancy request. The second team which was an inpatient multidisciplinary one including the 2 above specialists in addition to at least an Anesthesiologist or Intensivist /Radiologist/Pediatrician-Neonatologist was responsible for the therapeutic management once the mother was hospitalized. Almost all positive or highly suspected cases were hospitalized. In rare cases, outpatient management of pregnant mothers with COVID-19 was depended on the joint decisions of Obstetrician\& Gynecologist and Infectious diseases specialist.

Pharmacologic management

For outpatient cases antiviral treatment was added (Hydroxy chloroquine or Chloroquine phosphate). Inpatient treatment composed of

- Hydroxy chloroquinesulfate or Chloroquine Phosphate

- $\quad$ Kaletra

- In case of using Atazanivir/ritonavir, Hydroxy chloroquine sulfate for the complete duration of medical treatment was also prescribed.

\subsubsection{New items were added in this version}

- $\quad$ Multidisciplinary management team in hospitals was introduced.

- $\quad$ 24/7/30 virtual support of medical university multidisciplinary team by National Committee especially for ICU admitted mothers was announced.

\subsection{Third version of flowchart was released on 2020.04 .29}

Based on the evidences and reported maternal morbidity due to COVID-19 cardiomyopathy in pregnancy, cardiologic considerations were introduced in the third edition: 
- A Cardiologist was added to the Multidisciplinary Team (National /University/Hospital levels); moreover, the conditions that need Cardiology Consultation as well as indications of Echocardiography were described.

- Defining home-based care of infected/suspected mothers

- Screening of asymptomatic cases coming for vaginal Delivery or cesarean section

- Determining of gestational age limit for prescribing Corticosteroids for fetal lung maturation

Summary of 3 versions related to flowcharts and performed measures during the outbreak of COVID-19 are shown in Table 1.

Table 1 Summary of the performed measures during the outbreak of COVID-19 by the Maternal Health Office.

\begin{tabular}{|c|c|c|}
\hline Editions & date & measures \\
\hline 1 & 2020.2 .25 & $\begin{array}{l}\text { Providing a guideline for maternal care during COVID19 Pandemic } \\
\text { Screening of attended mothers in health centers with temperature measurement and } \\
\text { referring febrile cases to a physician } \\
\text { Identification of maternal screening modalities ( symptomatic cases /Asymptomatic) } \\
\text { Coordination with the ambulances to transfer infected pregnant women to the hospital } \\
\text { Emergency dispatch protocols } \\
\text { Virtual prenatal follow ups and canceling all childbirth preparation classes for pregnant } \\
\text { women } \\
\text { Using virtual modalities to train and inform mothers about prevention during outbreak } \\
\text { of COVID19 }\end{array}$ \\
\hline 1 & 2020.2 .26 & $\begin{array}{l}\text { Extracting maternal phone numbers from Health Electronic System and connecting them } \\
\text { via virtual pathways for follow ups } \\
\text { Distributing protocols for personal protective equipment (PPE) in Pregnancy } \\
\text { Introducing the designated hospitals to the Health Centers and mothers } \\
\text { Establishment of an Infectious Triage for all pregnant women entering the hospital } \\
\text { Designing a triage flowchart for maternal obstetrics /and or COVID19 complications } \\
\text { Observing the level of health services related to midwifery and neonatal cares at hospital } \\
\text { Emphasizing on perinatal regionalization } \\
\text { organizing a consultant committee composed of anesthesiologists, } \\
\text { obstetricians/gynecologists, Infectious diseases specialists, and midwives to supervise } \\
\text { the process of hospitalization, the time of termination of pregnancy and the kind of } \\
\text { delivery } \\
\text { Distributing protocols of maternal health committees at university level and national } \\
\text { level } \\
\text { Redesigning National Maternal Mortality Surveillance System with omission of outdoor } \\
\text { verbal autopsy and emphasizing on the finalization of maternal mortality cases } \\
\text { surveillance up to } 1 \text { month after each death } \\
\text { Sharing experience between medical universities through a regional video conferences } \\
\text { Distributing PPE Protocols for Care Givers }\end{array}$ \\
\hline 2 & 2020.3 .1 & $\begin{array}{l}\text { Inviting the Radiology Association for contributing actively in the National Maternal } \\
\text { Health Committee }\end{array}$ \\
\hline 2 & 2020.3 .2 & $\begin{array}{l}\text { Reducing Antenatal visits from } 8 \text { to } 4 \text { times }(6-10,28-30,31-34 \text { and } 37 \text { weeks) } \\
\text { Designing an Electronic Reporting System for suspicious or infected COVID19 maternal } \\
\text { cases (Inpatients /Outpatients) }\end{array}$ \\
\hline 2 & 2020.3 .3 & $\begin{array}{l}\text { Providing a triage flowchart regarding to referred pregnant women to the hospital } \\
\text { Medical treatment of COVID-19 infected pregnant women (in \& outpatients) } \\
\text { Making decision about the treatment, medication, hospital admission and time of } \\
\text { discharge by the medical team composed of obstetricians/perinatologist and infectious } \\
\text { diseases specialists }\end{array}$ \\
\hline
\end{tabular}




\begin{tabular}{|l|l|l|}
\hline 2 & 2020.3 .19 & $\begin{array}{l}\text { Distributing a protocol correlated to disinfecting of ultrasound probes and equipments } \\
\text { as well introducing a program to reception department how to minimize the virus spread }\end{array}$ \\
\hline 2 & 2020.3 .20 & $\begin{array}{l}\text { Updating the flowcharts related to hospitalization of the referred pregnant women } \\
\text { Determining the indications of radiography examinations, standard exposure doses and } \\
\text { set up for pregnant women } \\
\text { updating treatment protocol for admitted and outpatient pregnant women } \\
\text { Performing diagnostic virology tests } \\
\text { aOrganizing a medical treatment team composed of obstetricians/perinatologist, } \\
\text { internal medicine specialist/pulmonologist, pediatrician/neonatologist, and infectious } \\
\text { diseases specialist to prioritize medical measures through the first 6 hours of } \\
\text { hospitalization } \\
\text { - Establishment of a national scientific consultant team responding to all medical } \\
\text { universities (24 hours) }\end{array}$ \\
\hline 3 & $\begin{array}{l}\text { Determining the indications and instructions for heart consultation } \\
\text { Determining the instructions of Betametasone administration for pregnancies } \\
\text { threatened by preterm birth } \\
\text { Updating the treatment protocol for admitted and outpatient infected pregnant women } \\
\text { Adding cardiologist and radiologist consultants to the hospital treatment team }\end{array}$ \\
\hline
\end{tabular}

\subsection{Fourth version of flowchart released on 2020.05 .22}

\subsubsection{In this step, several items were considered and added as follows:}

- $\quad$ Defining different stages of the Disease (Mild, Moderate, Severe, and Critical)

- $\quad$ Increasing 02 Saturation tolerance limit in pregnancy from $93 \%$ to $95 \%$

- $\quad$ Considering mild-stage cases with systemic diseases as the high risk patients

- $\quad$ Dividing inpatient from outpatient triage protocol

- $\quad$ Defining self-isolation criteria and time of the end point of self-isolation

- $\quad$ Determination of Test-based and symptomatic-based approaches to quarantine period

- $\quad$ Revising pharmacologic Management;

- $\quad$ Outpatient pharmacologic treatment includes Hydroxy Choloroquine or Chloroquine Phosphate for at least 5 days and at most for 10 days exclusively indicated for high risk cases based on Infectious diseases specialist Prescription - $\quad$ Inpatient management includes Kaletra and Atazanavir /Ritonavir.

The final protocol is attached as Fig. 1.

\subsubsection{In this protocol, all details related to each situation were described as bellow;}

In Outpatient cases, minimum requirements for home care were:

- Maternal as well as her relatives' ability to monitor her condition

- $\quad$ Presence of a health care giver less than 65 years old in their household

- $\quad 24$ hours access to medical health services in cases of arising emergency conditions like Severe Dyspnea, disturbed consciousness, chest pain

- $\quad$ Separated rooms

- $\quad$ Avoidance of close contact with more than 65 years old people in household

- $\quad$ Separating bathrooms

Special conditions to be followed:

- PPE access for everyone in close contact with the patient

- Daily disinfecting of all contact surfaces

- Minimal presence in common spaces with the others, banning patient visits

- Use of medical masks and daily change of it

- Hands hygiene rules to be followed

- Good ventilator condition in the house

- Adequate access to food and medicines

- Knowing what to do and where to go in cases of emergency conditions 
Indications of cardiologic consultation in suspected/Confirmed COVID-19 maternal cases were:

1. Previous or present history of the cardiologic diseases like valvular heart diseases, congenital cardiac diseases, cardiomyopathies, past cardiac surgeries or cardiac interventional procedures, ischemic heart diseases, ETC

2. Presence of any predisposing risk factors related to cardiac diseases like diabetes, Hypertension, Smoking, Obesity (BMI equal or more than 30), Twin Pregnancy, Preeclampsia, and so on

3. Progressive increase of Troponin Level to more than twice basis or $99 \%$ in 48 hours from the time of hospitalization, in pregnant Suspected/Confirmed COVID -19, troponin should be tested at the time of hospitalization first

4. Presence of one or more of the following conditions :

- Pericardial effusion

- Pleural effusion

- Dominancy of Paracardiac or central involvement

- Vavular /Coronary and or pericardial calcification

- Obvious cardiomegaly in chest CT Scan or disproportionate cardiomegaly in pregnancy in CX Ray

5. Obvious EKG Changes:

- Sustained supra ventricular or ventricular arrhythmia

- AV block

- Significant ST-T changes

- Prolonged QT

6. Pathologic cardiac murmur or any changes in the heart auscultation in comparison to primary findings

7. Heart rate per minute of less than 60 or more than 120

8. Hypotension with following criteria:

- Systolic pressure equal or lower than 90 in a normotensive patient

- $40 \mathrm{~mm} \mathrm{Hg}$ reduction of systolic blood pressure in a hypertensive patient

9. Pro BNP laboratory Titrage more than 450

10. Generalized Edema

11. Symptoms cannot be justified only by COVID -19

Echocardiography indications were also:

1. Shock State

2. PAC Isolated or Isolated PVC JNew arrhythmia except

3. Significant cardiomegaly in CT scan

4. Pericardial effusion in CT scan

5. Pro BNP laboratory Titrage more than 450

6. And new EKG changes like Block or ST-T changes Significant

7. Generalized Edema

8. Aggravation not justifiable by COVID-19 patients with known cardiac diseases and symptomatic

Asymptomatic cases should be screened. All those coming to labor wards should be screened regarding to:

- $\quad$ History of contacts in recent 2 weeks

- $\quad$ Temperature, symptoms like cough, throat ache, dyspnea, malaise, rhinorrhea, Olfactory or Taste disturbances

In case of any positive answers to any or all of these questions the patient will be referred to infectious diseases specialist for further evaluation.

Below Figure 1, 2, and 3 represents the fourth version of flowchart associated with management of pregnancy complicated with COVID -19. 


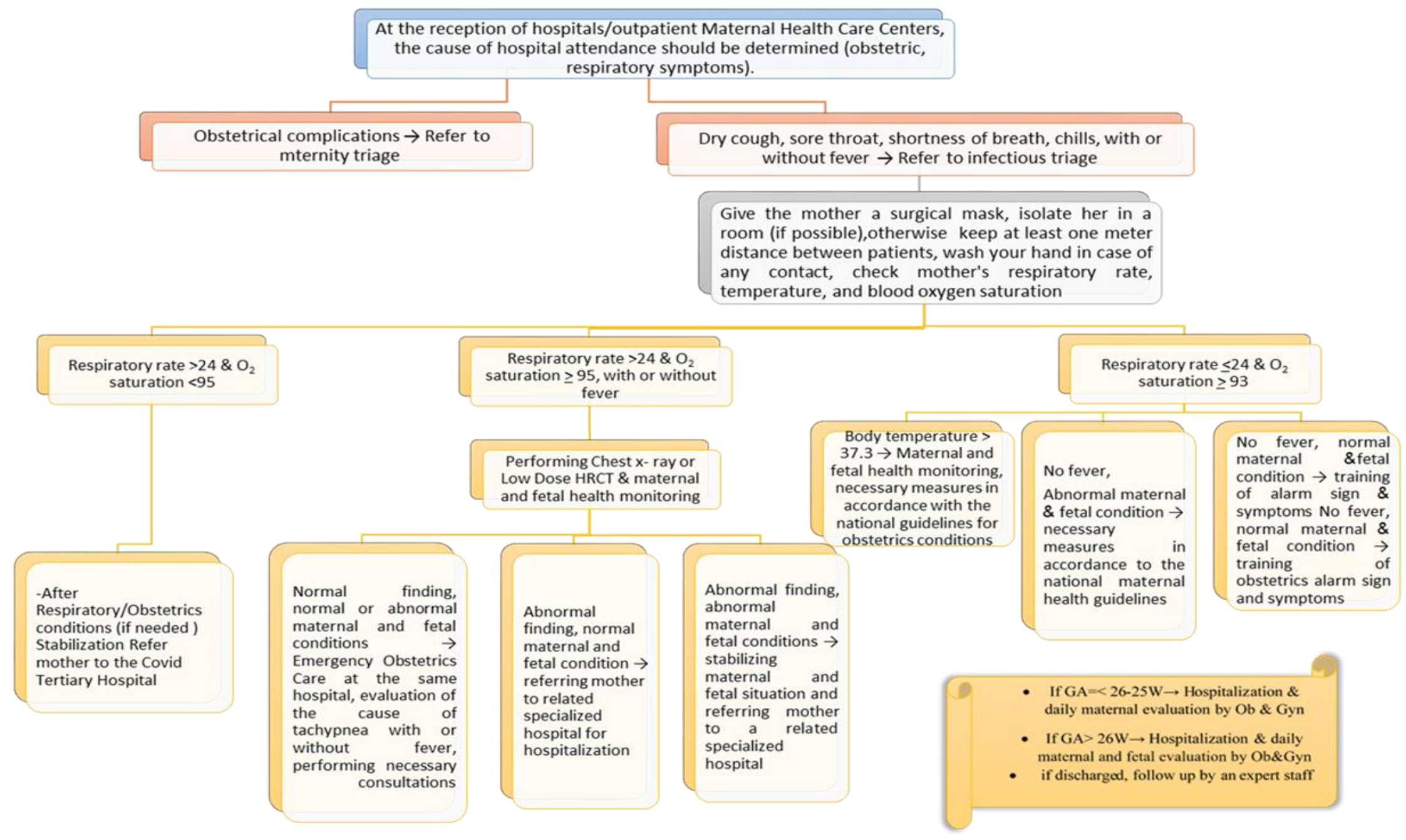

Figure 1 COVID-19 Guidance for Maternity Triage 


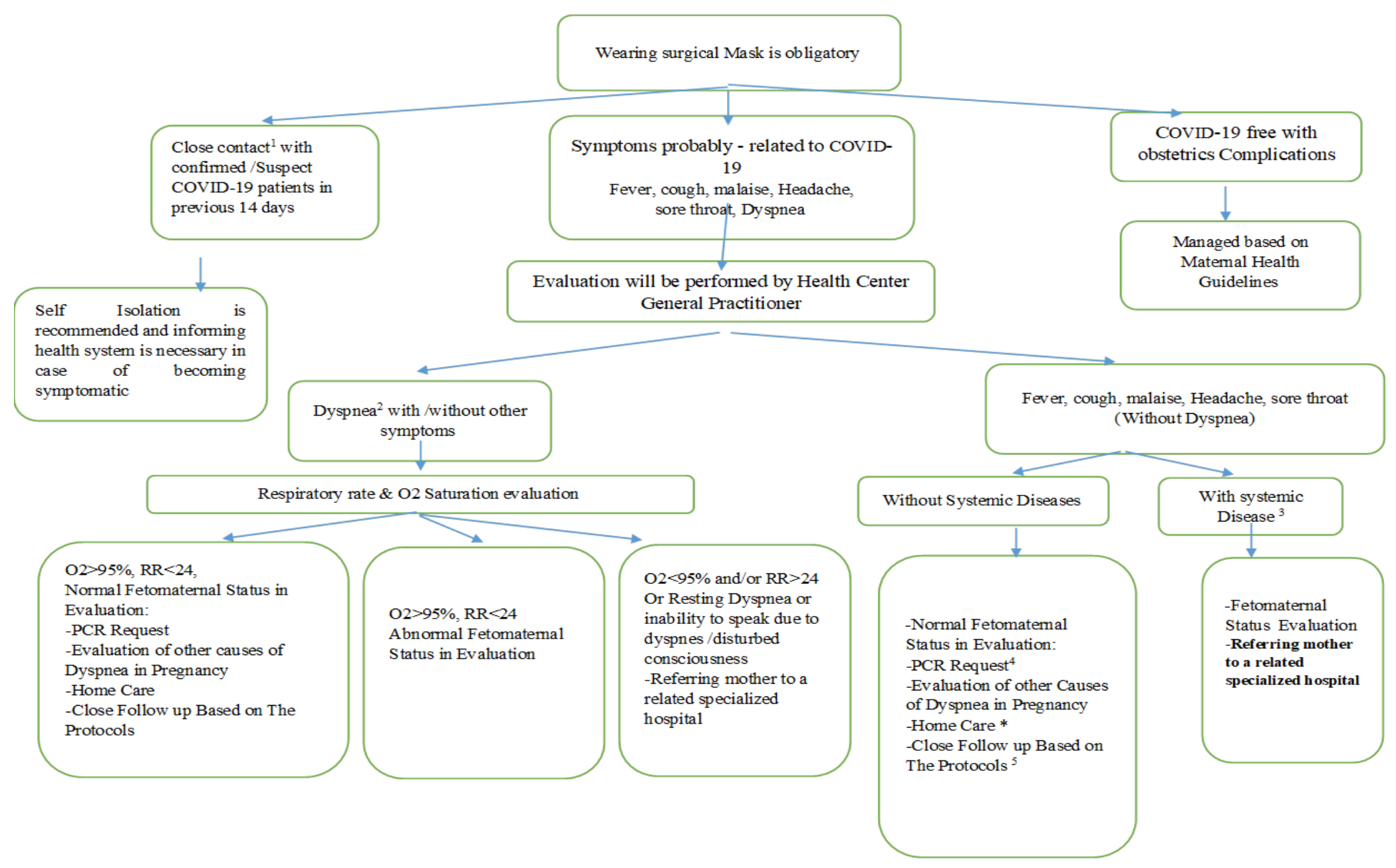

Figure 2 Outpatient COVID -19 screening in pregnancy protocol 


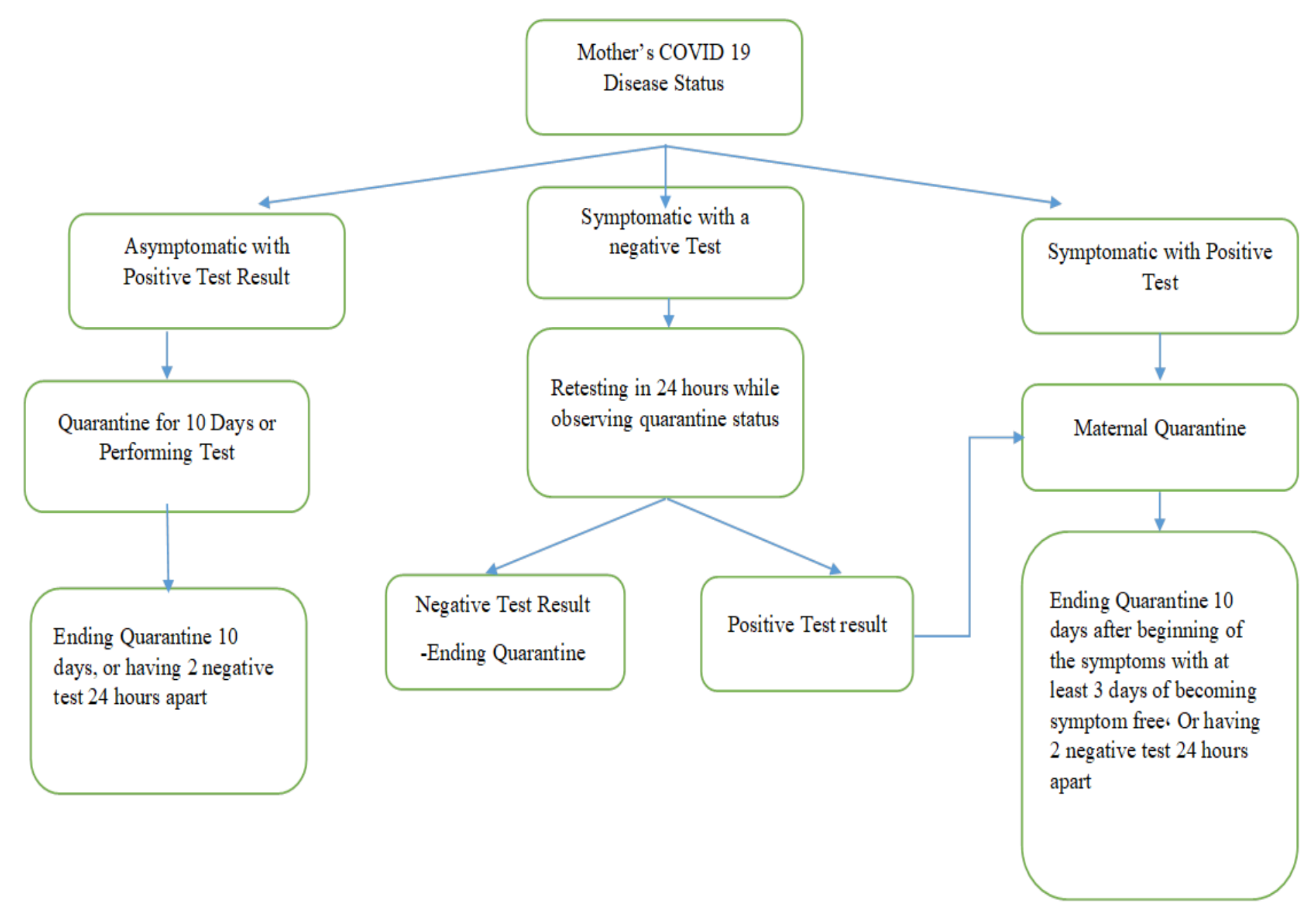

Figure 3 Possible-confirmed COVID-19 ending Home-quarantine protocols for pregnant mothers 
If GA= <26-25W - Hospitalization \& daily maternal evaluation by Ob \& Gyn; If GA> 26W- Hospitalization \& daily maternal and fetal evaluation by $\mathrm{Ob} \& \mathrm{Gyn}$. If discharged, follow up by an expert staff.

1. Less than 2 meters distance from Suspected/Confirmed cases for more than 15 minutes while not using mask or in contact with his/her body secretions. 2. Disease range of covid-19 is from mild-Intermediate-Severe - Critically ill cases in need of Intensive cares 3. Systemic diseases which impose at higher risks are Diabetes, Hypertension, Renal Diseases, Pulmonary Diseases, Cardiovascular Diseases, HIV infection and other immunocompromising Diseases 4. PCR in Suspected Cases is performed for at least twice with a 24 hours interval. PCR result may be false Negative and for this reason symptomatic cases should be followed 5. Following Up of Outpatient cases (Whether discharged from hospitals or primarily cared in Home) should take place at least on $1^{\text {st }}$ day, $4^{\text {th }}, 7^{\text {th }} 10^{\text {th }}$ of home care via High Risk Technical Officer (a midwife specially assigned for this duty in Public health Centers)

\section{Conclusion}

Regarding to Triage system for COVID-19 in pregnancy, there were paucity of data and documents. We used adult triage flow chart as the prototype and adapted it to pregnancy situation. Then based on the more evidences and more and more experiences, this triage system were updated.

\section{Compliance with ethical standards}

\section{Acknowledgments}

Present study was approved by of Corona Virus Scientific Committee in Iran Ministry of Health and Medical Education. We acknowledge all contributors. Special thanks to Obstetrics \& Gynecology, Radiology, Intensive care and Anesthesiology Scientific Associations.

\section{Disclosure of conflict of interest}

The authors declare that they have no conflict of interest.

\section{References}

[1] Rasmussen SA, Smulian JC, Lednicky JA, Wen TS and Jamieson DJ. (2020). Coronavirus Disease 2019 (COVID-19) and Pregnany: What obstetricians need to know. American Journal of Obstetrics and Gynecology, In Press.

[2] Mardani M and Pourkaveh B. (2020). A Controversial Debate: Vertical Transmission of COVID-19 in Pregnancy. Arch Clin Infect Dis, 15(1), 102286.

[3] Chen H, Guo J, Wang C, Fan L, Xuechen Y, Wei Z, Jiafu L, Dongchi Z, Dan X, Qing G and Jing L. (2020). Clinical characteristics and intrauterine vertical transmission potential of COVID-19 infection in nine pregnant women: a retrospective review of medical records. Lancet, 395, 809-815.

[4] Qiao J. (2020). What are the risks of COVID-19 infection in pregnant women? The Lancet, 395, 10226.

[5] Royal College of Obstetricians \& Gynaecologists. Coronavirus (COVID-19) Infection in Pregnancy: Information for healthcare professionals.

[6] Zhu H, Wang L, Fang C, Sicong Peng S, Zhang L, Chang G, Shiwen X and Wenhao Z. (2020). Clinical analysis of 10 neonates born to mothers with 2019-nCoV pneumonia, Transl Pediatr, 9(1), 51-60.

[7] Report: COVID-19 Transmitted to Babies in Utero.

[8] Vivanti AJ, Vauloup-Fellous C, Prevot S, Do Cao J, Benachi A and De Luca. (2020). Transplacental transmission of SARS-CoV-2 infection. Nat Commun, 11, 3572.

[9] Hantoushzadeh S, Shamshirsaz A, Aleyasin A, Seferovic MD, Kazemi Aski S and Arian SE. (2020). Maternal death due to COVID-19. Am J Obstet Gynecol, In Press.

[10] Irshad A, Ahamed A, Nashwa E and Bee KT. (2020). First Covid-19 maternal mortality in the UK associated with thrombotic complications. Br J Haematol, ID: covidwho-291290. 
World Journal of Advanced Research and Reviews, 2020, 07(03), 051-061

[11] Mascio D, Khalil A, Saccone G, Rizzo G, Buca D and Liberati M. (2020). Outcome of coronavirus spectrum infections (SARS, MERS, COVID-19) during pregnancy: a systematic review and meta-analysis. American Journal of Obstetrics \& Gynecology MFM, 2(2), 100107. 